and (ii) in the case of rhombic primitive period-parallelogram

$$
\sigma_{2 s}\left(c^{\prime}\right)=(-1)^{s}(2 c)^{2 s} \sigma_{2 s}(c) \quad\left(c c^{\prime}=\frac{1}{4}\right) .
$$

The computation has been carried out up to $2 s=50$ with adequate guarding figures provided for $\sigma_{4}$ and $\sigma_{6}$. The values are then rounded off to $16 \mathrm{D}$. Individual check is made on the last two coefficients by direct summation of the double series. The results up to $2 s=20$ are shown in Tables 1 and 2 . In Table 2 , the values of $\sigma_{4}$ and $\sigma_{6}$ are not included, which may be found in reference 2 . The complete table is deposited in the UMT file in the office of the journal.

The author is grateful to the National Council on Science Development, Taiwan, China, for support of the work.

Institute of Mathematics

Academia Sinica

Taiwan, China

1. C.-B. Ling, "Evaluation at half periods of Weierstrass' elliptic function with rectangular primitive period-parallelogram," Math. Comp., v. 14, 1960, p. 67-70. MR 22 * 1061 .

2. C.-B. LING \& C.-P. TSAI, "Evaluation at half periods of Weierstrass' elliptic function with rhombic primitive period-pargllelogram," Math. Comp., v. 18, 1964, p. 433-440.

3. E. T. Copson, An Introduction to the Theory of Functions of a Complex Variable, Clarendon Press, Oxford, 1935, p. 359-362.

\title{
A Method for the Computation of the Error Function of a Complex Variable
}

\section{By Otto Neall Strand}

Abstract. This paper presents a method of computing erf $z \equiv(2 / \sqrt{ } \pi) \int_{0}^{z} e^{-u^{2}} d u$, where $z$ is complex. It is shown that $\operatorname{erfc} z \equiv 1-\operatorname{erf} z$ has no zeros in the right-hand half plane. An estimate of $|\operatorname{erfc} z|$ is derived.

The error function of a complex variable, denoted by erf $z$, is defined by the equation erf $z=(2 / \sqrt{ } \pi) \int_{0}^{z} e^{-u^{2}} d u$, where $z$ is complex. This function arises in many problems of physics and engineering. Several methods [1], [2], [3] have been devised for the computation of erf $z$ and closely-related functions, and several tabulations [4], [5], [6] have been made. The method to be described below has two features which make it relatively simple to use: (1) the phase enters in a simple explicit manner; and (2) the major portion of the computation consists of the accumulation of two series of positive terms for which each term (after the first) may be calculated by a simple recursion without the use of transcendental functions. For the particular FORTRAN double-precision programs which were written for comparison, the average computing time for the method of this paper was found to be approximately $\frac{7}{10}$ of that for Salzer's first method [7] for an equally-spaced grid of points throughout the region defined by $0<|z|<6.6$ and $0 \leqq \arg z<\pi / 2$. The relative difference between results from the two methods was less than $10^{-13}$ throughout this region.

Since the relations erf $\left(-z_{0}\right)=-\operatorname{erf} z_{0}$ and $\overline{\operatorname{erf}\left(z_{0}\right)}=\operatorname{erf}\left(\overline{z_{0}}\right)$ may always be employed to reduce the computation to one involving $z_{0}$ in the first quadrant, the

Received May 1, 1964. 
following derivation is restricted to the computation of erf $z_{0}$, where $z_{0}=x_{0}+i y_{0}$, $x_{0}>0$ and $y_{0} \geqq 0$. The case $x_{0}=0$ is not covered by this method.

By Cauchy's theorem:

$$
\operatorname{erfc} z_{0} \equiv 1-\operatorname{erf} z_{0}=\frac{2}{\sqrt{ } \pi} \int_{c} e^{-u^{2}} d u,
$$

where $C$ is the hyperbola $x y=x_{0} y_{0}=v_{0}$ for which the integrand has constant phase, described in the direction of increasing $x$ from $x=x_{0}$ to $x=\infty$. Reduction of the line integral to definite integrals gives the result

(2) $\operatorname{erfc} z_{0}=H_{1} \cos 2 v_{0}-y_{0} H_{2} \sin 2 v_{0}+i\left[-H_{1} \sin 2 v_{0}-y_{0} H_{2} \cos 2 v_{0}\right]$, where

$$
\begin{aligned}
& H_{1}=\frac{2}{\sqrt{ } \pi} \int_{x_{0}}^{\infty} \exp \left[-\left(x^{2}-\frac{v_{0}^{2}}{x^{2}}\right)\right] d x, \\
& H_{2}=\frac{2 x_{0}}{\sqrt{ } \pi} \int_{x_{0}}^{\infty} \frac{1}{x^{2}} \exp \left[-\left(x^{2}-\frac{v_{0}^{2}}{x^{2}}\right)\right] d x .
\end{aligned}
$$

We expand the integrands of $H_{1}$ and $H_{2}$ in series as follows:

$$
\begin{aligned}
& H_{1}=\frac{2}{\sqrt{ } \pi} \int_{x_{0}}^{\infty} e^{-x^{2}}\left(\sum_{n=0}^{\infty} \frac{\left(v_{0}\right)^{2 n}}{n ! x^{2 n}}\right) d x, \\
& H_{2}=\frac{2 x_{0}}{\sqrt{ } \pi} \int_{x_{0}}^{\infty} e^{-x^{2}}\left(\sum_{n=0}^{\infty} \frac{\left(v_{0}\right)^{2 n}}{n ! x^{2 n+2}}\right) d x .
\end{aligned}
$$

Since all terms in the series are positive, term-wise integration can be justified by the Lebesgue Monotone Convergence Theorem [8], so that

$$
\begin{aligned}
& H_{1}=\sum_{n=0}^{\infty} \gamma_{n} v_{0}^{2 n}, \\
& H_{2}=x_{0} \sum_{n=0}^{\infty}(n+1) \gamma_{n+1} v_{0}^{2 n},
\end{aligned}
$$

where

$$
\gamma_{n}=\frac{2}{n ! \sqrt{ } \pi} \int_{x_{0}}^{\infty} \frac{e^{-x^{2}}}{x^{2 n}} d x, \quad n=0,1,2, \cdots .
$$

Since $\gamma_{0}=$ erfc $x_{0}$, it can be obtained from existing methods. To obtain the other $\gamma$ 's we integrate $\gamma_{n+1}$ by parts to obtain

$$
\gamma_{n+1}=\frac{2}{(2 n+1) \sqrt{ } \pi}\left[\frac{e^{-x_{0}^{2}}}{(n+1) ! x_{0}^{2 n+1}}-\frac{\sqrt{ } \pi}{n+1} \gamma_{n}\right], \quad n=0,1,2, \cdots .
$$

The method of computation consists of computing the series (5), where the coefficients are obtained recursively by (7). The values of $H_{1}$ and $H_{2}$ are then substituted into (2) to obtain erfe $z_{0}$, from which erf $z_{0}$ is obtainable by (1).

Although the following results are of some interest, they do not pertain directly to the method of computation. By (2),

$$
\left|\operatorname{erfc} z_{0}\right|=\sqrt{ }\left(H_{1}{ }^{2}+y_{0}{ }^{2}{H_{2}}^{2}\right) \text {. }
$$


Therefore erfe $z$ has no zeros in the right-hand half plane. This property is evident in examining the contour charts due to Laible [9]. It can be shown [10] that

$$
\int_{x_{0}}^{\infty} e^{-x^{2}} d x<\frac{e^{-x_{0} 2}}{2 x_{0}} \quad \text { for } x_{0}>0 \text {. }
$$

Therefore

$$
\begin{aligned}
& H_{1}<\frac{\exp \left(y_{0}{ }^{2}-x_{0}{ }^{2}\right)}{x_{0} \sqrt{ } \pi}, \\
& H_{2}<\frac{\exp \left(y_{0}{ }^{2}-x_{0}{ }^{2}\right)}{x_{0}{ }^{2} \sqrt{ } \pi} .
\end{aligned}
$$

Combination of (8) with (9) gives the following estimate for the absolute deviation of erf $z_{0}$ from 1 :

$$
\left|\operatorname{erfc} z_{0}\right|<\frac{e^{y_{0}^{2}-x_{0}^{2}}}{x_{0} \sqrt{ } \pi} \sqrt{ }\left(1+y_{0}^{2} / x_{0}^{2}\right) .
$$

This estimate may be useful in some cases to determine if erf $z_{0}$ may be approximated by 1 with sufficient accuracy.

National Bureau of Standards

Boulder, Colorado

1. J. B. Rossen, "Theory and application of $\int_{0}^{z} e^{-x^{2}} d x$ and $\int_{0}^{z} e^{-p^{2} y^{2}} d y \int_{0}^{y} e^{-x^{2}} d x$." Part 1, Methods of Computation, Mapleton House, Brooklyn, N. Y., 1948. MR 10, 267.

2. H. E. SAlzER, "Formulas for calculating the error function of a complex variable," $M T A C$, v. 5, 1951, p. 67 . MR 13, 989; 1140 .

3. J. Kestin \& L. N. Persen, "On the error function of a complex argument," $Z$. Angew. Math. Phys., v. 7, 1956, p. 33-40. MR 17, 968.

4. B. D. Fried \& SAMUel D. ConTe, The Plasma Dispersion Function. The Hilbert Transform of the Gaussian, Academic Press, New York, 1961. MR 24 * B1958.

5. K. A. Karpov, Tables of the Function $w(z)=e^{-z^{2}} \int_{0}^{z} e^{x^{2}} d x$ in a Complex Region, Izdat. Akad. Nauk SSSR, Moscow, 1954. (Russian) MR 16, 749.

6. V. N. FADDEeva \& N. M. Terent'ev, Tables of Values of the Function $w(z)=$ $e^{-z^{2}}\left(1+2 i \pi^{-1 / 2} \int_{0}^{z} e^{t^{2}} d t\right)$ for Complex Argument, (English translation), Mathematical Tables Series, v. 11, Pergamon Press, London, 1961. MR $22 * 12740$.

7. H. E. SALZER, op. cit., Formulas (6) and (7), p. 68 .

8. M. E. MUNROE, Introduction to Measure and Integration, Addison-Wesley, Cambridge, Mass., 1953. MR 14, 734 .

9. T. LAIBLe, "Höhenkarte des Fehler-integrals," Z. Angew. Math. Phys., v. 2, 1951, p. 484-487. MR 13, 495.

10. William Frller, An Introduction to Probability Theory and Its Applications, Vol. I, Wiley, New York, 1950, Lemma 2, p. 131 (p. 166 of 2nd ed.). MR 12, 424. 